\title{
1 An ancestral genomic sequence that serves as a nucleation site for de 2 novo gene birth
}

\author{
Nicholas Delihas
}

Department of Microbiology and Immunology, Renaissance School of Medicine, Stony 
A short non-coding sequence present between the gamma-glutamyltransferase 1 (GGT1) and gamma-glutamyltransferase 5 (GGT5) genes, termed a spacer sequence has been detected in the genomes of Mus musculus, the house mouse and in Philippine tarsier, a primitive ancestral primate. It is highly conserved during primate evolution with certain sequences being totally invariant from mouse to humans. Evidence is presented to show this intergenic sequence serves as a nucleation site for the initiation of diverse genes. We also outline the birth of the human lincRNA gene BCRP3 (BCR activator of RhoGEF and GTPase 3 pseudogene) during primate evolution. The gene developmental process involves sequence initiation, addition of a complex of tandem transposable elements and addition of a segment of another gene. The sequence, initially formed in the Old World Monkeys such as the Rhesus monkey (Macaca mulatta) and the baboon (Papio anubis), develops into different primate genes before evolving into the human $B C R P 3$ gene; it appears to also include trial and error during sequence/gene formation. The protein gene, GGT5 may have also formed by spacer sequence initiation in an ancient ancestor such as zebrafish, but spacer and GGT5 gene sequence drift during evolution produced a divergence that precludes further assessment.

Key words: de novo gene birth; long intergenic non-coding RNAs (lincRNA); gene evolution; transposable elements; chromosomal tandem repeats

\section{Author summary}

For a number of decades researchers have been interested in how genes evolve and a number of mechanisms of gene formation have been defined. This manuscript describes a different process of gene formation, that of a small DNA sequence that does not code for a gene but serves as a nucleation site for the initiation of de novo gene formation. This non-coding DNA sequence appears to have been in existence for about hundred million years or more and has formed the basis for the birth of diverse genes during evolution of the primates. The questions of how and why new genes are

53 born are important in terms of revealing how organisms, especially primates, progress

54 to greater complexity during evolution; the question of "how" is particularly relevant to 
55 the creation of biological information ab initio during prebiotic and early cellular

56 evolution.

\section{Introduction}

Protein genes are created by varied processes that include gene duplication [1-5], retrogenes [6] and de novo formation [6-12]. With respect to the latter, Knowles and McLysaght [8] first reported that several human protein-coding genes arose by a de novo mechanism, and Wu et al [9] identified 60 protein-coding genes that are also born by a de novo process. Less has been reported on origins of long intergenic noncoding RNA (lincRNA) genes. However, some examples are lincRNA genes created from pseudogenized protein genes [13] and lincRNA family genes formed by gene duplication [14]. In addition, the formation of a new human lincRNA gene by transcriptional readthrough has been reported. The work of Rubino et al [15] shows that by use of the transcriptional apparatus of an existing gene and transcriptional readthrough to a small intergenic sequence that represents a functional unit, a new gene is created. This new gene is thought to participate in regulation of the immune system. This study has similarities to the work of Shiao et al [16] concerning de novo acquired 3' UTRs that may play important functions of retrogenes, and that of Stewart and Rogers [17] in terms of the recruitment of non-coding sequences with chromosomal rearrangements and the resultant formation of new protein genes. Thus far, the creation of lincRNA genes appears similar to that of protein genes.

Here we describe a different process of de novo gene birth. It was previously thought that the lincRNA FAM247 family gene sequence may serve as a nucleation site for new gene birth [18]. However, outlined here is a non-coding DNA sequence, termed a spacer sequence that is situated between the gamma-glutamyltransferase 1 (GGT1) and gamma-glutamyltransferase 5 (GGT5) genes. It is present in the rodent house mouse (Mus musculus) and ancestral prosimian primitive primates such as Philippine tarsier (Carlito syrichta) and is evolutionarily conserved in the genomes of all higher primates. It consists of less than $4000 \mathrm{bp}$, and in many species can contain small sections of the FAM247 sequence. We show that this spacer sequence is a nucleation site for new gene formations. We find that the 3 ' ends of spacers are sites for the in initiation of de novo sequence growth with the creation of diverse genes during primate evolution. In addition, the chimpanzee genome provides an example of the combination of spacer sequence duplication and de novo gene formation at the duplicated genomic locus, which is partly analogous to chromosomal rearrangements and the resultant generation of de novo genes described by Rogers and Stewart [17]. Eight

93 experimentally and/or computationally determined genes have been detected that stem 
94 from spacer sequences during primate evolution and all sequences starts with the

95 elongation of the FAM247 sequence.

Also presented here are the evolutionary formations of two human long non-coding RNA genes, the lincRNA gene BCRP3 (the BCR pseudogene 3 ), and the $F A M 247 A, C, D$, long intergenic RNA family genes, and propose a model for the formation of the BCRP3 sequence in the Rhesus monkey. With these genes, a trial and

101 error process to produce the complete sequence appears to have occurred in several ancestral primates. We also discuss the presence of a significant length of conserved

103 transposable elements (TEs), Alu/LINE TE tandem repeats found in the BCRP3 sequence. These tandem repeats pose interesting questions of origin and function. Aside from non-coding RNA genes, it is possible that the GGT5 protein gene, whose sequence also begins with an FAM247 sequence and is found in non-mammalian ancestors, may also have formed via spacer sequence initiation. The zebrafish genome may be an ancestral example where GGT5 was born, but the spacer sequence significantly diverged during evolution, which makes further assessment of spacer involvement in GGT5 formation difficult.

\section{Results}

\section{Properties of spacer sequences}

Computational alignment and search programs were used to analyze genomes of primates and other species. The primitive early primate, Philippine tarsier genome was found to display a small genomic spacer sequence (2872 bp) situated between the protein genes GGT1 and GGT5 (Fig. 1). The spacer sequence between GGT1 and

120 GGT5 in the house mouse Mus musculus genome is also shown. A large expansion at

121 this genomic region occurred during primate evolution as the Rhesus monkey sequence

122 between genes GGT1 and GGT5 shows an increase in size to 216,200 bp; this

123 sequence expansion is on chr10 and the sequence is also found inverted with

124 chromosomal rearrangements (Fig. 1). The chimpanzee genome continued this

125 genomic expansion with an increase to $343,330 \mathrm{bp}$; the human genome maintained

126 most of this sequence but decreased by $\sim 10 \%$. The spacer sequence between GGT1

127 and GGT5 of the primitive primate Philippine tarsier is found in the higher primates

128 linked to the GGT1 gene after genomic expansion. The expanded genomic regions

129 contain duplicated sequences that have provided for the formation of a number of new

130 genes or family of genes. However, of significance, the GGT1-spacer sequence 3' ends 
131 are found to be focal points, or nucleation sites where diverse genes and/or sequences

132 originate from the GGT1-spacers in various primate species, the spacer 3' end serving

133 as the starting point for growth of new sequences and/or genes.

Primate genomic expansion between GGT1 and GGT5

Mus musculus (house mouse)

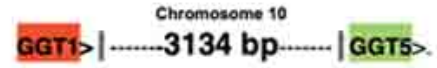

Carlito syrichta (Philippine tarsier)

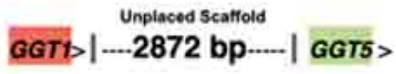

Macaca mulatta (Rhesus monkey)

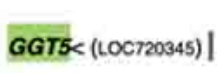

Chromosome 10

Gark
Approximate

evolutionary time

million years ago (MYA)

90 MYA

50 MYA

25 MYA GGTS 6 MYA
134

135

136

137

138

139

140

141

142

143

144 Fig. 2a depicts several diverse genes in genomic regions that follow the 3' ends of the

145

146

147

148

149

150

151

Fig. 1. The spacer region/genomic lengths between GGT1 and GGT5 in various species. The house mouse and Philippine tarsier (member of ancestral primates) are in the top two drawings. The lengths of genomic regions between genes GGT1 and GGT5 in the higher primates are shown below. The chromosomal region is inverted in Rhesus and other primates. Chromosomal locations are also shown above the schematics. The approximate evolutionary time is on the right. Genomes of these species were analyzed from the NCBI data base (https://www.ncbi.nlm.nih.gov). GGT1-spacer sequences, and these genes are present in different species. Additionally, in humans, gene duplication of the GGT-spacer motif gives rise to both GGT-related family genes and the FAM247 lincRNA family genes (Fig. 2b). In terms of mechanism of initiation and growth of newly formed sequences from spacer 3' ends, we do not know the source of the FAM247 template or the primer for DNA synthesis, or even if there is a template involved in new DNA synthesis.

Pan troglodytes (chimpanzee)

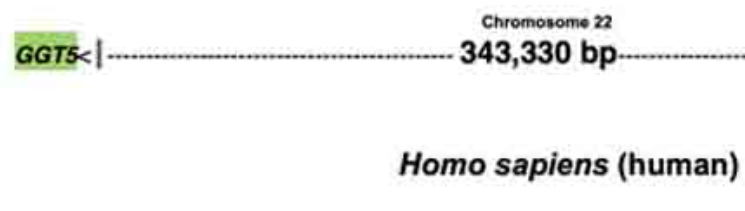

GGTS<1 $\quad 338,610$ bp IGGTS OMYA 


\section{a. Diverse genes linked to the GGT1-spacers in different species}

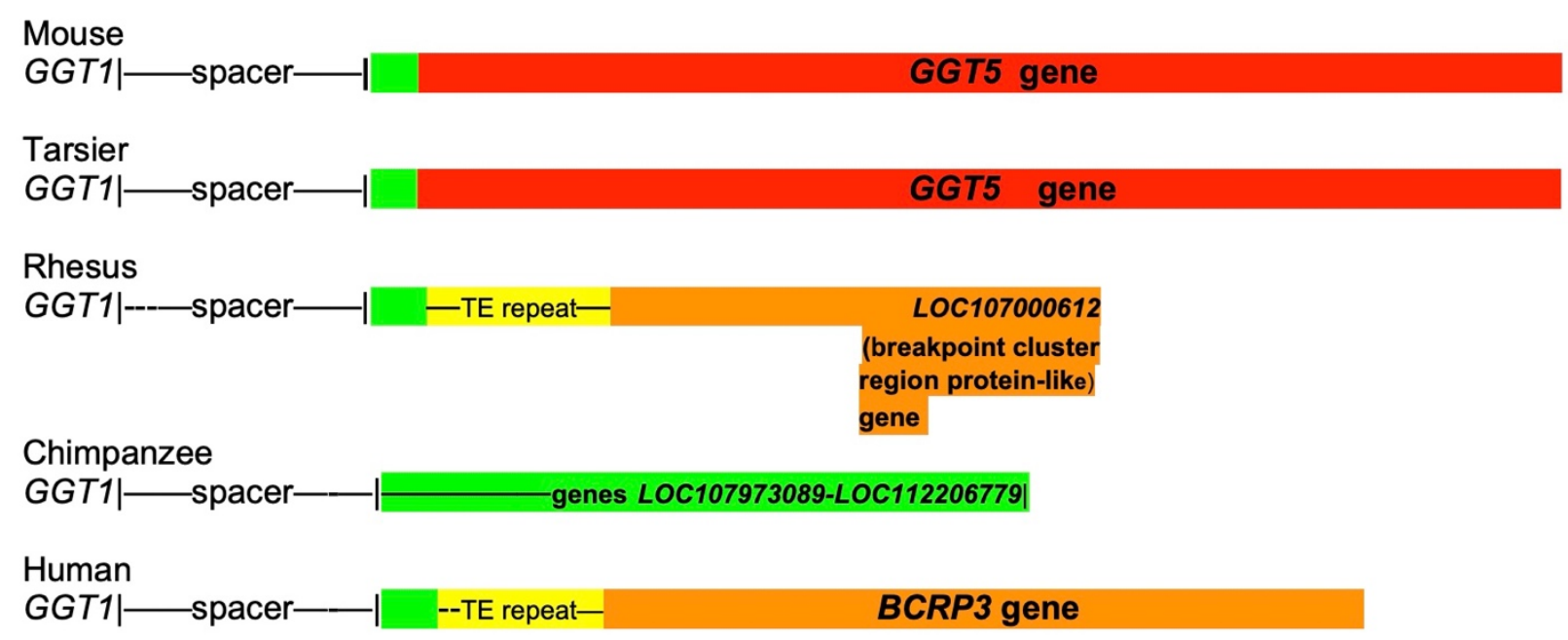

Segmental duplications in human chr22

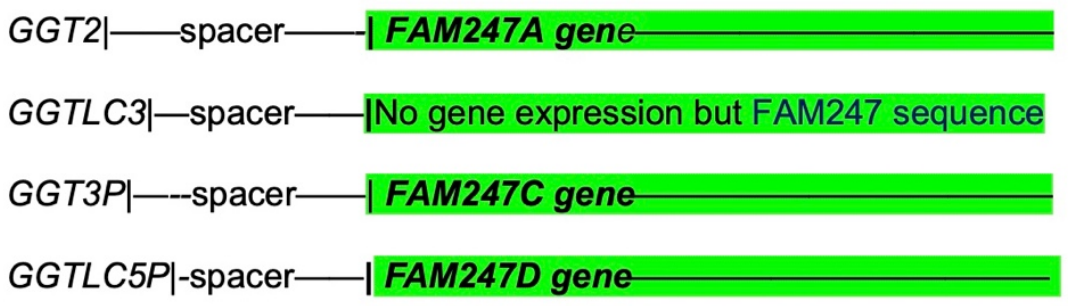

Fig. 2. a. Diverse genes (GGT5, LOC107973089, LOC112206779, LOC107000612, BCRP3 and FAM247) present in different species that stem from GGT1-spacer sequence 3' ends. The FAM247 sequence (highlighted in green) is partially or totally present in all genes and/or sequences linked to spacers. The yellow highlighted regions represent Alu/LINE TE tandem repeat arrays. The tan areas contain sequences of the $B C R$ gene. b. Diagrammatic representation of the spacer sequences that lead to gene sequences, The green highlighted regions represent FAM247 sequences present in different genes that start close to the 3' ends of spacers. GGT-related genes are highlighted in re and BCR-related in tan. There are FAM247 5 ' end sequences present in some spacers, e.g., mouse and tarsier but not depicted in the diagram. b. The GGT-spacer-FAM247 gene family sequences present in different segmental duplications in human chr22 [14]. There appears to be no transcript expression from the FAM247 sequence associated with the GGTLC3-spacer, although the sequence has $99.4 \%$ identity with lincRNA gene FAM2347A and contains the entire FAM247A sequence [15]. GGT family genes GGT2, GGTLC3, GGT3P, and GGTLC5P are protein or pseudogenes that developed at duplicated loci. 25bp and $354 \mathrm{bp}$ of FAM247 are not in the GGT5 sequences of the mouse and Tarsier, respectively, and 34 and 36 base pairs of the 5' end of FAM247 are not in the BCRP3 sequence GGT5 genes of Rhesus and humans, respectively. 
171 The 3' ends of the mouse and tarsier spacer sequences are defined by the start of the 172 GGT5 gene (Fig. 1). Because of sequence expansion in the higher primates, the GGT5 173 gene locations cannot be used to define the ends of spacers. However, we have used 174 the presence of other genes or the FAM247 sequence where there are no gene 175 annotations to define the 3' end of spacers in the higher primates. The 5' half sequence 176 of FAM247 is present in all genes/sequences that stem from spacer 3' ends; the term

177 FAM247 is used throughout the manuscript to denote the lincRNA FAM247A gene

178 sequence or part of it. The presence of the 5' end FAM247 sequence is helpful in

179 estimating the 3 ' ends of the spacers from species where there are no gene annotations 180 immediately following the spacer but where the FAM247 sequence is present, e.g., in 181 the baboon, gibbon and orangutan genomes.

Table 1 shows a high conservation of the overall bp sequence of spacer sequences between the primates, and a $56 \%$ sequence identity between the mouse and human spacers. In addition, the spacer 3 ' regions display blocks of totally invariant sequences amongst the primates and the mouse (highlighted in light blue, Fig. 3). The functions of these conserved sequences are not known, but because of their invariance over evolutionary time, they may function to initiation gene sequence from the spacer 3 ' end.

Table 1. GGT1-associated spacer sequences and percent identity

191 between species

192

\begin{tabular}{|lc|}
\hline Species spacer sequences & \%ldentity* \\
\hline human spacer (GGT1-BCRP3) & 100.00 \\
\hline chimpanzee spacer (GGT1-FAM247) & 98.45 \\
\hline & \\
\hline Rhesus spacer (GGT1-FAM247) & 89.86 \\
\hline tarsier spacer (GGT1-GGT5) & 72.94 \\
\hline & \\
\hline mouse spacer (GGT1-GGT5) & 56.20 \\
\hline
\end{tabular}

193 *relative to the human spacer sequence

195 There are spacer sequences between GGT1 and GGT5 in the zebrafish and opossum 196 genomes, but these have significantly diverged in base pair sequence and do not 
197 display conserved sequence blocks; they have not been included in the comparisons in 198 Table 1 or in Fig. 3.

200 Additional sequence conservation is within the spacer 5' end region, and it is shown in

201 the alignment of the 5' end spacer gene sequences from all species considered (S1

202 Fig. a.).This alignment also has an added sequence, the NCBI sequence termed: GGT1

203 RefSeq, Homo sapiens gamma-glutamyltransferase 1 (GGT1) NG_008111.1 (website:

204 Homo sapiens gamma-glutamyltransferase 1 (GGT1), RefSeqGene on chromosome

205 22). Note that the GGT1 RefSeq contains the entire GGT1 gene sequence but also

206 includes regions beyond the 5' and 3' ends of the gene, for example, the (GGT1),

207 RefSeqGene extends 2010 bp beyond the GGT1 gene 3' end. The sequence alignment

208 between spacer sequences from different species, with the extended 2010 bp sequence

209 included, show a similarity in sequence from position 1-1451 bp of the (GGT1)

210 RefSeqGene with sequences of the 5' ends of the spacers, particularly with sequences

211 from the Rhesus to human. Of significance, the distantly related prosimian primitive

212 primate gray mouse lemur spacer shows a particularly high identity (84\%) with part of

213 the human (GGT1) RefSeqGene 3' end sequence (S1 Fig. b); thus, a segment of the 5'

214 region of the spacer sequence shows a high evolutionary conservation that spans $\sim 55$

215 million years. The 2010 bp sequence, which follows the GGT1 gene 3' end, makes up a

216 large portion of the spacer sequence in humans. 
GGT1.END-GGT5.end.75422027-75453034.mouse GGT1.END-GGT5.START.75422027-75425161.mouse GGT1 .end-GGT5 . beginining. Philippine.tarsier.ref FAM2 4 7.LOC105372935.ref.human

GGT1.end-FAM2 47.start. Rhesus.ref.

GGT1.end-start.BCRP 3.. human.ref

GGT1end-LOC749026.end.7456450-7520130.chimp GGT1.end-FAM247.start.chimp.re

GGT1.END-GGT5.end. 75422027-75453034.mouse GGT1.END-GGT5.START. 75422027-75425161.mouse GGT1.end-GGT5 . beginining.Philippine.tarsier.ref FAM2 47.LOC105372935.ref.human

GGT1.end-FAM247.start. Rhesus.ref .

GGT1.end-start.BCRP 3. .human.ref

GGT1end-LOC749026.end.7456450-7520130.chimp GGT1.end-FAM247.start.chimp.ref

GGT1.END-GGT5 .end. 75422027-75453034.mouse GGT1.END-GGT5.START . 75422027-75425161.mouse GGT1.end-GGT5 . beginining.Philippine.tarsier.ref FAM2 4.LOC105372935.ref.human GGT1.end-FAM2 47 .start. Rhesus.ref.

GGT1.end-start.BCRP 3. .human. ref

GGT1end-LOC749026.end.7456450-7520130.chimp

GGT1.end-FAM247. start. chimp.ref

GGT1.END-GGT5 .end. 75422027-75453034.mouse GGT1.END-GGT5.START.75422027-75425161.mouse GGT1.end-GGT5 . beginining.Philippine.tarsier.ref FAM2 47. LOC105372935.ref. human

GGT1.end-FAM247. start. Rhesus.ref.

GGT1 .end-start. BCRP 3. . human. ref

GGT1end-LOC749026.end.7456450-7520130.chimp

GGT1.end-FAM247.start.chimp.ref catcacatttccaatggcactgggactgaggagtctttgggtggtgttggggcagcaggg 3045 catcacatttccaatggcactgggactgaggagtctttgggtggtgttggggcagcaggg 3045 cgccaaggcctcaagcatattcagcggggatgggac----------------------- 2429

0

caccaagttctcctgcacattgggacagtgtgaccctgggctctggttagtg--gcaggt 2816 caccaagttctcctgcacattgcgacagtgtgaccetgggctctggcgggca--gtaggt 3770 caccaagttctcctgcacattgcgacagtgtgacctgggctctggcgggeg--gtaggt 3776 caccaagttctcctgcacattgcgacagtgtgaccetgggetctggcgggcg--gtaggt 3776

caggccatgggatcaactggegatggaagagttaacagcggcagctggctcttctcaaga 3105 caggccatgggatcaactggcgatggaagagttaacagcggcagctggctcttctcaaga 3105 -------------cacggcagcaagggagttaaccgcagcagctggctc--ctgta-g 2471 (1) 0

ggggccttgggtcctaccagcagtgagggagttagca-cagcagctggctc--ctctagg 2873 ggggcetttggacctaccagcagtgagggagttaaca-cagcagctgactc--ctctagg 3827 ggggcctttggacctaccagcagtgagggagttaaca-cagcagctgactc--ctctagg 3833 ggggcctttggacctaccagcagtgagggagttaaca-cagcagctgactc--ctctagg 3833

Start of mouse GGT5 gene sequence, highlighted in red

aaaaaaaactccctgtaga---------tgcctggcttgcctccagggttgagcctcggg 3157 aaaaaaaactcctgtaga---------tgcctggctt----------------------- 3135 caaagaaaactcccc-cagacgctttgctgcctggccttccgccagggetgagaa--cag 2528

gaaggaaactcccttcagacactttggtgcctggcctcctgccaggaacaagca---gg 2930 caaggaaaactccctcagacgetttgctgcetggcetcctgccagcaacaagca---gg 3884 caaggaaaactccctcagatgctttgctgcctggcctcctgccagcaacaagca---gg 3890 caaggaaaactcccetcagatgctttgctgcctggcctcctgccagcaacaagca---gg 3890

Start of FAM247 sequence, highlighted in green

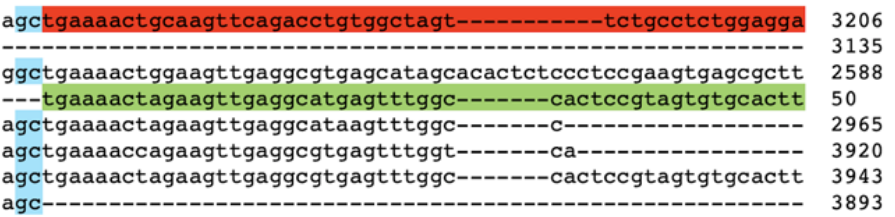

Fig. 3. Small segment of alignment of spacer sequences showing the start of the FAM247 sequence and conserved sequences. Alignment of five spacer sequences from mouse, tarsier, Rhesus, chimpanzee and humans. Only section of the spacer 3' terminal ends, the start of the mouse GGT5 and the start of FAM247 sequences are shown. Spacer terminal ends: mouse, at 3161 bp; tarsier, 2872 bp ; Rhesus, 2933 bp; chimpanzee, 3893 bp; human, 3920 bp. Light blue highlighted, conserved sequence blocks that are conserved in all species analyzed,. Green highlighted, the start of FAM247 sequence. Red highlighted, start of GGT5 gene sequence in the mouse genome. In the higher primates, since GGT5 is distal to GGT1, the start of the GGT5 sequence can not be used to define the 3 ' ends of the spacers and the FAM247 sequence has been used. The lengths of spacer 3 ' ends vary between species. However, the spacer end of the chimpanzee is shown, i.e., position 3893 bp of the chimpanzee sequence from GGT1.endFAM247.start.chimp.ref, which ends before the FAM247 sequence begins. In humans, the BCRP3 gene contains the FAM247 sequence but starting with position 33 of the FAM247, with positions 1-32 bp of FAM247 present in the human spacer, therefore we have defined the start of the BCRP3 gene sequence as the human spacer 3 ' end. The alignment of the complete sequences used is in S2 Fig.

241 Of the three experimentally determined genes that are linked to spacer sequences, i.e., 242 GGT5, BCRP3 and the FAM247A-D gene family, GGT5 is the most difficult to analyze 
in terms of mechanism of formation. The gene is present in the genome of zebrafish, an

244 ancestral vertebrate species that predate the rodents and primates. However, the gene

245 bp and protein aa sequences have significantly diverged over evolutionary time.

246 Although there are small blocks of aa acid sequences such as ${ }_{280}$ PPPPAGGA ${ }_{287}$ in the

247 zebrafish GGT5b aa sequence that are totally conserved in all species analyzed, i.e.,

248 zebrafish, opossum, mouse and all primates, the overall zebrafish GGT5b gene aa

249 sequence shows an identity of only $48 \%$ relative to the human sequence, showing a

250 poor aa sequence similarity with the other GGT5 genes. However, there is a continuum

251 of decline of aa identity relative to the human gene aa sequence during evolution that

252 shows a continuous sequence drift for this gene (S3 Fig). The aa sequence blocks of

$253100 \%$ aa identity, such as the one shown above, may be related to important functional

254 roles of these invariant segments from the GGT5 protein. Included in S3 Fig. is the

255 GGT5 aa sequence of the opossum (Monodelphis domestica, gray short-tailed

256 opossum), which is approximately 175 MYA in evolutionary age and thus predates the

257 rodents. Addition of the opossum aa sequence aids in the assessment of the

258 evolutionary changes in GGT5 aa sequence and pattern of change and supports the

259 continuum of evolutionary changes observed. From the GGT5 aa sequences that have

260 been analyzed, the data suggest that the GGT5 genes from zebrafish to humans are

261 evolutionarily related.

262

263 Evidence was presented to show that GGT5 exon1 consists entirely of the FAM247

264 sequence in humans, primates and the mouse, but the FAM247 presence in zebrafish

265 was uncertain [18]. Here we show evolutionary changes of GGT5 exon1 aa sequences,

266 with the opossum exon1 aa sequence included (Fig. 4); this helps show the trend in loss

267 of conserved aa found with evolutionary time, but also supports the evolutionary

268 conservation of certain aa positions, which are found to be highly biased in terms of the

269 presence in different regions of the peptide chain (Fig. 4). There is a substantial loss of

270 conserved aa residues in the first two thirds of the sequence, but a stability at the

271 carboxyl terminal end of the exon1 sequence where a majority number of aa residues

272 do not change from primates, rodents, opossum and zebrafish (Fig. 4). Thus, although

273 the overall percent identity of GGT5 exon1 aa sequences from zebrafish and opossum

274 compared to that of humans is poor, the invariant aa positions of exon 1 and their highly

275 biased locations in the peptide chain suggest an FAM247-type sequence also forms

276 exon1 of zebrafish and opossum GGT5 genes. Development of the zebrafish GGT5

277 gene's 5' end sequence may have begun with the FAM247 sequence, but how the

278 GGT5 sequence was extended and matured to its full sequence during its birth, either in

279 zebrafish or another early ancestor, is not known. 


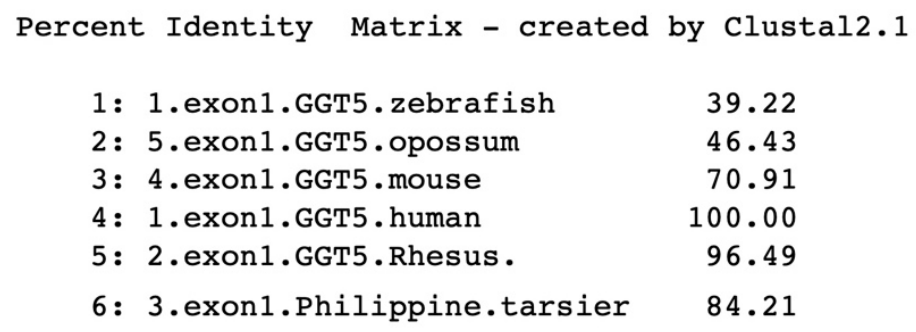

exon1.GGT5b.zebrafish 5. exon1.GGT5.opossum

4. exon1.GGT5.mouse

1. exon1.GGT5. human

2.exon1.GGT5.rhesus.

3.exon1.Philippine.tarsier
MAKSQSRRCCFCLLALVC--TAAIICICILFSK-----QKCDFTRAAVSADSLMCSDIGR 53 MARPGGRAVCLILLAAGL--LAAIIAAACTLGRAAATCPAASYRTAAVAADTPRCSAIG- 57 MAWGHRATVCLVLLGVGLGL--VIVVLAAVLSPRQASCGPGAFTRAAVAADSKICSDIG- 57 MARGYGATVSLVLLGLG--LALAVIVLAVVLSRHQAPCGPQAFAHAAVAADSKVCSDIG- 57 MARGCGATVGLVLLGLG--LALAVIVLAVVLSRHQAPCGPQAFAHAAVAADSKVCSDIG- 57 MAWGCRAI ISLVLLGLGLGLALVIIVLAVVLPRHQAPCGPQAFAHAAIAADSKVCSDIGR 60 ** $\quad: * * . \quad$ : : . : $\quad$ : **::**: ****

Fig. 4. Alignment of amino acid sequences of exon 1 from GGT5 proteins from various species. Top. The percent identities between the human exon 1 and other species. Bottom. Amino acid sequences alignment showing tinvariant aa residues with *. Aligned by Clustal2.1 (https://www.ebi.ac.uk/Tools/msa/clustalo/).

\section{BCRP3: Formation of the BCRP3 sequence in Rhesus}

In terms of gene expression, the human $B C R P 3$ gene produces one transcript that is expressed primarily in the testes (NCBI https://www.ncbi.nlm.nih.gov/gene/?term=Homo+sapiens+BCRP3) [19]. Structurally, $B C R P 3$ consists of approximately the 5' half of $F A M 247 A$ gene sequence, an Alu/LINE TE tandem repeat array, and a copy of a segment of the $B C R$ (the BCR activator of RhoGEF and GTPase) gene sequence [18] (Fig. 5a). The BCRP3 gene offers an interesting picture of how a gene sequence was created and evolved in primates over evolutionary time. Using sequence blast searches, the earliest detection of the BCRP3 sequence is in the Old World monkeys, the Rhesus monkey and baboon. In Rhesus, the BRCP3 sequence is found in chr10, linked to the GGT1-spacer at its 3' end, however the BCRP3 sequence has differences; primarily, it is shorter compared to the human BCRP3 (S4 Fig). The Rhesus BCRP3 sequence contains the 5' half of the FAM247 sequence (with $88 \%$ identity compared to the human $B C R P 3$ ), significant differences in the Alu/LINE TE tandem repeat array (Table 2) and a sequence segment of the $B C R$ gene sequence with a significantly shorter BCR component compared to the human BCRP3 gene (Fig. 5a). 
There are no genes annotated at the start of the BCRP3 sequence, but there is a gene stemming from the 3' end of the Rhesus BCRP3 sequence, the computationally derived protein gene LOC107000612 annotated as breakpoint cluster region protein-like (Fig. $5 \mathrm{a})$; it comprises $2889 \mathrm{bp}$ and is homologous to the human BCRP3 3 ' end segment with $90 \%$ identity. Thus, a putative gene stems from the Rhesus BCRP3 sequence but the major portion of the BCRP3 sequence has no annotations, and the protein gene LOC107000612 greatly differs from the human lincRNA BCRP3 gene.

A model for the formation of the BCRP3 sequence in Rhesus is described below. Fig. $5 b$ graphically shows the proposed model of BCRP3 formation.

\section{Model of the formation of the BCRP3 sequence in Rhesus monkey}

1. Initiation and growth of the BCRP3 sequence begins at the 3' end of the spacer with the elongation of the FAM247 sequence up to FAM247 position 5955 bp (Fig. 5b, section 1). The spacer may provide signals to initiate synthesis of the FAM247 sequence.

2. An array of contiguous Alu/LINE tandem repeats, other TEs, and AT simple repeats are added to the FAM247 sequence (Fig. 5b, section 2). Table 2 shows the TE tandem repeats. A search for a copy of a similar Alu/LINE TE tandem array in other parts of the Rhesus genome was negative. This is in contrast to the human Alu/LINE TE tandem array in the $B C R P 3$ gene where an almost identical TE Alu/LINE array is present in the human IGL locus [18]. How the TE tandem repeats were added to the growing sequence in Rhesus is not known. However, there are significant differences with TE insertions and simple repeats between the Rhesus and human TE tandem arrays (Table 2). Also, the tandem repeat in human BCRP3: AluSg- AluSx1- AluSg- L1MEg- AluSx1- L1MEg- AluSg4 consists of a nearly perfect tandem repeat array with no extraneous base pairs between repeating TEs, which is not the case for the Rhesus array (S5 Fig). This suggests a de novo formation of TE arrays with each species.

3. The Rhesus $B C R$ (BCR activator of RhoGEF and GTPase) is a large gene of 133735 bp. A small section (6682 bp) of the 3' end of BCR is copied and transferred to the growing Rhesus BCRP3 sequence (Fig. 5b, section 3). The segment of the $B C R$ gene present in the Rhesus monkey is homologous to the human $B C R P 3$ gene sequence (with $82 \%$ identity) but its length is shorter than that in the human BCRP3 gene (Fig. 5a). A copy of part the Rhesus $B C R$ gene sequence may have been transferred to the growing Rhesus BCRP3 sequence linked to the Rhesus GGT1-spacer. There is also a 
347 partial copy of the Rhesus $B C R$ sequence present in the Rhesus IGL locus, but it is

348 unlikely the source of the BCR sequence in the Rhesus BCRP3 as the BCR fragment in

349 the IGL locus is not long enough.

350

\section{a. Components of the partial BCRP3 sequence in the Rhesus monkey compared to human BCRP3 gene}

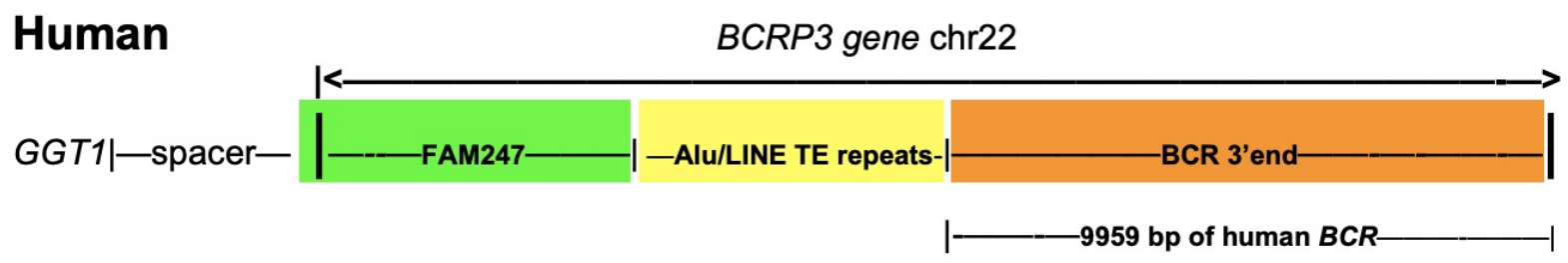

Rhesus $\quad$ BCRP3 sequence chr10

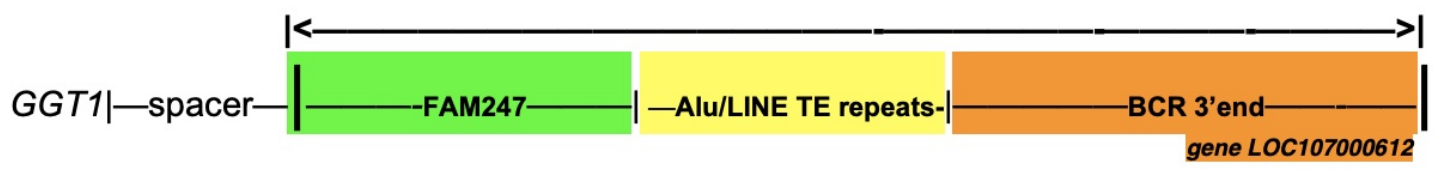

$\mid-6682$ bp of Rhesus $B C R-\mid$

\section{b. Proposed formation of BCRP3 sequence in Rhesus}

\section{Initiation and growth of FAM247 sequence}

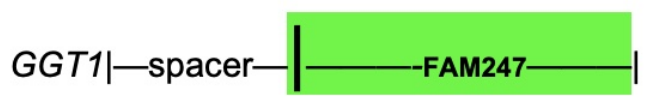

।

\section{Addition of Alu/LINE TE repeats}

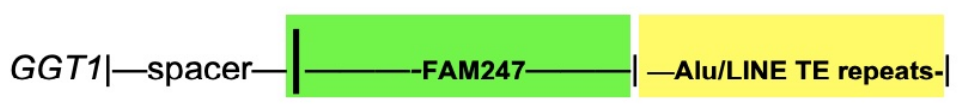

\section{Addition of BCR 3' segment}

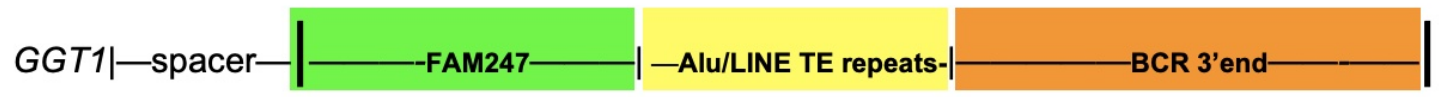

352 Fig. 5. a. A schematic of the composition of the BCRP3 sequence in Rhesus compared to that

353 of the human_BCRP3_gene. b. The proposed formation of the BCRP3 sequence in Rhesus,

354 with three steps that involve initiation of synthesis and sequence growth, followed by addition of 
a complex of TE motifs and ending with addition of a segment of a gene from another part of the genome.

Table 2. Alu/LINE TE tandem arrays in BCRP3 sequences of primates*

\begin{tabular}{|llll|}
\hline Human & Rhesus & Baboon & Chimpanzee \\
\hline L1MEg & L1MEg & L1MEg & L1MEg \\
\hline MER4E1. & (AT)n & AluY & MER4E1 \\
\hline AT)n. & AluSx & MER4E1 & (AT)n \\
\hline (ATATACACAC)n & AluSx & (AT)n & AluSg \\
\hline (AT)n & AluSx & AluSg & AluSx \\
\hline AluSg & L1MEg & AluSx & AluSx1 \\
\hline AluSx1 & AluY & AluSx1 & L1MEg \\
\hline AluSg & AluSz6 & L1MEg & AluSx1 \\
\hline L1MEg & L1MEg & AluSx1 & L1MEg \\
\hline AluSx1 & AluSg4 & L1MEg & AluSg4 \\
\hline L1MEg & (TTAT)n & AluSx & L1MEg \\
\hline AluSg4 & AluSx & (T)n & AluSx \\
\hline L1MEg & AluYRb3. & L1MEg & AluSx \\
\hline AluSx & AluSx & AluSx & L1MEg \\
\hline AluSx & AluSx & AluSx & AluSx \\
\hline L1MEg & L1MEg & L1MEg & L1MEg \\
\hline AluSx & AluSx & AluYRa1 & AluJb \\
\hline L1MEg & L1MEg & L1MEg & L1MEg \\
\hline AluJb & AluJb & AluSg & FLAM_A \\
\hline L1MEg & L1MEg & L1MEg & MADE1 \\
\hline FLAM_A & AluJb & L1MEg & A-rich \\
\hline MADE1 & AluY & FLAM_C & AluY \\
\hline (A)n & L1M2 & A-rich & L1M2 \\
\hline AluY & & L1MEg & \\
\hline L1M2 & & & \\
\hline DIM & & \\
\hline
\end{tabular}


BCRP3: The process of formation of the BCRP3 sequence in the baboon, gibbon and orangutan

The baboon is classified as part of the Old World monkeys and is related to the Rhesus monkey, but diverged 2 MYA [20]. It has partially developed the BCRP3 sequence at its genomic GGT1-spacer locus but did not progress as far as the Rhesus in sequence development. It has the FAM247 sequence up to FAM247 position 5955 bp at 92\% identity with the human BCRP3 gene and compared with the Rhesus BCRP3 sequence at $88 \%$ and has the repeat Alu/LINE TE array (Table 2). The tandem repeats of the baboon are more similar to the human repeats than to those of the Rhesus, but missing in the baboon TE tandem array are an Alu, and MADE1 that are present in the human array at the 3' end (Table 2). Significantly however, the baboon BCRP3 sequence differs from that of the Rhesus in that it does not have a copy of the BCR gene segment (S6 Fig.) and in terms of similarity of the partial sequence, it is closer to the human $B C R P 3$. In addition, there are no genes annotated at the locus where the homologous partial BCRP3 sequence resides in the baboon genome. Thus, there is no apparent explanation for synthesis of the partial BCRP3 other than a failed attempt to synthesize a more complete BCRP3 type sequence or produce a sequence that can encoded a gene.

Fig. 6 summarizes the variety of sequences that stem from the 3' ends spacer sequences in different species of the superfamily Hominoidea. The gibbons (Nomascus leucogenys (northern white-cheeked gibbon) are part of the family Hylobatidae, a branch of the superfamily Hominoidea (that consists of the human-like apes and humans) but are the lesser apes or small apes. Their evolutionary appearance is 17MYA. Of major interest, at the GGT1-spacer locus, only part of the FAM247 sequence has formed up to FAM247 position 4467bp, which is shorter than the Rhesus FAM247 sequence at 5955 bp, but it displays a high identity with the human FAM247 sequence (95\%). In addition, at this chromosomal locus, the gibbon sequence does not have a Alu/LINE TE tandem array and does not have a copy of the BCR segment of the $B C R$ gene. There are no annotated genes that stem from the partial FAM247 sequence.

402 Thus, it appears to have initiated a partial human FAM247 gene sequence with a high

403 identity with the human FAM247 at the gibbon GGT1-spacer locus, but was

404 unsuccessful in completion of a full FAM247 sequence, the presumed end result. 
406 The gibbon genome, however, has formed an almost complete BCRP3 sequence, but

407 at another chromosomal locus, a GGT-spacer duplication locus that has the GGT2 408 gene-spacer sequence (not the GGT1-spacer) (Fig. 6a). Although it has a base pair 409 identity of $95 \%$ compared to the human $B C R P 3$, there are several gaps in the sequence 410 and one large additional sequence (3307bp) present in the gibbon BCRP3 sequence 411 that is not present in the human BCRP3 gene (S7 Fig). There are two genes annotated 412 within part of the BCRP3 sequence, the gibbon LOC115835989 breakpoint cluster 413 region protein-like and LOC115835847, the putative POM121-like protein 1 (Fig. 6a). It 414 appears the gibbon formed a sequence close to that of the human $B C R P 3$ but may 415 have used this sequence to form two genes of its own.

417 The orangutans are also part of the superfamily Hominoidea and are classified with 418 the great apes. The orangutan appeared evolutionarily about 9 MYA. Similar to the 419 baboon, the orangutan has formed only a part of the BCRP3 sequence at its GGT1420 spacer sequence locus and appears to have "regressed" in capacity to mature the 421 BCRP3 sequence compared to the Rhesus. The sequence includes the FAM247 422 sequence and the tandem TE repeat array, but does not have the BCR sequence that 423 forms the 3' end region of the Rhesus BCRP3 sequence (Fig. 6a) (S8 Fig.). In addition, 424 the partial sequence formed by the orangutan has several small sequence repeats that 425 may represent polymerase stuttering. It also has no putative genes that are annotated 426 within the FAM247-Alu/line TE tandem repeat sequence. Thus, the orangutan, which is 427 evolutionarily more advanced than the Rhesus monkey has not formed the BCRP3 428 sequence comparable to that of the Rhesus. Similar to the baboon, the orangutan may 429 have come to a "dead-end" in producing a more extended or complete BCRP3 430 sequence.

432 The Alu/LINE TE repeat region of the orangutan does have major differences in the 433 middle of the sequence compared to the human Alu/line TE tandem repeat. There are 434 insertions of three copies of an SVA_A retrotransposon and it is missing two LiMEg 435 elements (S9 Fig.). SVA insertions are known to affect function [21, 22]. The three 436 SVA_A retrotransposon insertions in the orangutan sequence may be related to an 437 inability to form a more complete BCRP3 sequence, however the baboon, which also 438 contains no BCR sequence, does not have retrotransposon insertions in its BCRP3 439 sequence; thus it is unlikely the retrotransposons are the cause of the partial sequence 440 in the orangutan. 
444 Similar to the gibbon, the chimpanzee genome also took an unexpected pathway with respect to BCRP3 sequence development where BCRP3 synthesis occurred at a different chromosomal locus from the GGT1 site of synteny, at a locus that represents a duplication of the GGT-spacer motif (Fig. 6a). This locus encodes a glutathione hydrolase light chain 2-like protein gene (LOC100610580) and it is 130,795 bp removed from the GGT1 chromosomal locus of synteny in the chimpanzee genome. There is a full sized BCRP3 sequence formed by the chimpanzee at this duplication site with a high identity, 98\% compared with the human BCRP3 sequence (S10 Fig.). The chimpanzee has the identical Alu/LINE TE array in its BCRP3 sequence as the human $B C R P 3$ gene except for differences in several subfamilies of Alus, and repeat sequences that are present in the human BCRP3 gene and not in the chimpanzee (Table 2). The major overall differences between the chimpanzee and human BCRP3 sequences are an AluY insertion in the human sequence and an AluSx insertion in the chimpanzee; these Alus are outside of the region containing the repeat Alu/LINE TEs. Thus, the chimpanzee, together with the gibbon, formed the complete the BCRP3 sequence as opposed to the baboon or orangutan, but the chimpanzee formed a sequence that is closer to the human $B C R P 3$ than that of the gibbon.

The duplication locus in the chimpanzee, which has the glutathione hydrolase light chain 2-like protein (LOC100610580) and the BCRP3 sequence, shows a computationally predicted gene annotated as LOC11220671, a breakpoint cluster region protein-like pseudogene (Fig. 6a). This gene is $5323 \mathrm{bp}$ in length and has a sequence that is homologous to positions $11710 \mathrm{bp}-17033 \mathrm{bp}$ of the human $B C R P 3$ gene; it thus has only about one quarter of the $B C R P 3$ gene sequence. The functions of both of the putative pseudogene LOC112206717 and the human pseudogene BCRP3 gene are unknown.

470 In humans, an inactive glutathione hydrolase 2 protein (LOC102724197) has been 471 annotated in an Unlocalized Scaffold region of chr22 (NT_187386.1). The NCBI transcript table shows 14 transcripts associated with LOC102724197. The inactive glutathione hydrolase 2 protein gene does have a linked spacer sequence but interestingly, it also has the complete FAM247 sequence instead of the BCRP3 related sequence that is found linked to chimpanzee LOC100610580, the glutathione hydrolase light chain 2-like protein (Fig. 6a and 6b). An FAM247 sequence may have formed de novo at this scaffold region of human chr22; alternatively, the FAM247 sequence at this locus may have originated by gene duplication. 
bioRxiv preprint doi: https://doi.org/10.1101/2022.01.12 475983; this version posted January 12, 2022. The copyright holder for this preprint (which was not certified by peer review) is the author/funder, who has granted bioRxiv a license to display the preprint in perpetuity. It is made available under aCC-BY 4.0 International license.

a.

Gibbon

BCRP3 sequence (complete sequence)

GGT2. |spacer| 5960 bp FAM247-Alu/LINE TE

LOC115835989 breakpoint cluster region protein-like

LOC115835847 the, putative POM121-like protein 1

Orangutan

BCRP3 sequence (incomplete sequence)

| GGT1 |spacer| | 5960 bp FAM247-Alu/LINE TEs| no BCR sequence

(3 SVA_A

insertions

2 LiMEg

Chimpanzee

Sequence
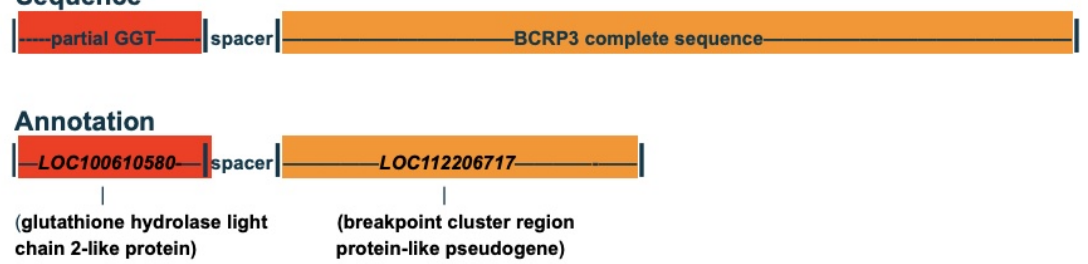

Human (BCRP3 gene at chr22 LCR22H)

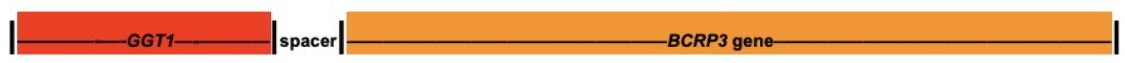

b.

Gibbon

$\prod_{- \text {GGT1 }}$ spacer $\mid 4468$ bp FAM247-no Alu/LINE TE- no BCR sequence incomplete FAM247 sequence

\section{Chimpanzee}

Sequence

|_GGT1____ $\mid$ spacer __ FAM247 complete sequence___

Gene annotations

|_GGT1__ | spacer| LOC107973089-LOC112206779

(protein genes)

Human chr22 LCR22D

-

FAM247A gene-

Human Unlocalized Scaffold region of human chr22 (NT_187386.1)

FAM247 complete sequence

(no gene annotations)

inactive glutathione hydrolase 2 protein 
Fig. 6. Formation of different sequences and/or genes at spacer 3 ' ends sequences from species of the superfamily Hominoidea. a. BCRP3 sequence present at loci that contain a duplication of the GGT-spacer motif showing diverse genes stemming from the BCRP3 sequence. $b$. The presence of the FAM247 sequence at different GGT-spacer loci in gibbon, chimpanzee and human genomes.

\section{FAM247: formation of complete sequence in chimpanzee and the gene in human}

Fig. $6 \mathrm{~b}$ shows a schematic of the FAM247 sequence that is present at the chimpanzee GGT1 locus. This sequence has a $97 \%$ identity with the human FAM247A lincRNA gene sequence and contains the entire length of the FAM247A gene sequence ( $\mathrm{S} 11$ Fig). The chimpanzee GGT1 linked spacer may have served as a nucleation site to initiate FAM247 synthesis, but unlike the synthesis of the BCRP3 sequence, there was continued synthesis of FAM247 until the complete FAM247 sequence was formed. There may be signal(s) directing the addition of TE tandem repeats to an FAM247 growing sequence, but in the absence of such signal(s), there may be continued FAM247 sequence growth. However, why there is a very partial FAM247 sequence in the baboon genome is not understood. Part of the chimpanzee FAM247 sequence is annotated as two genes, LOC107973089, and LOC112206779, and both are termed uncharacterized protein genes. Thus, the computationally derived protein genes in the chimpanzee differ from the human lincRNA genes where both types of genes stem from the same DNA sequence, or part of it.

Human genome chr22 has at least four copies of the FAM247 sequence and three genes that represent the FAM247 A,C, and $D$ lincRNA family (Fig. 2b) [15]. The evolutionary relationship of the chimpanzee FAM247 sequence to the human FAM247 long non-coding RNA gene family is unclear. It is not known if there was de novo synthesis of the FAM247 gene in humans and subsequent duplication of the linked sequence by segmental duplications (see Babcock et al for chr22 duplications [23]), or that the sequence was inherited from the chimpanzee and the FAM247 sequence developed in humans into the FAM247 gene with minor mutations and an association with a transcriptional apparatus [24]. With respect to the GGT protein family genes and human chr222 segmental duplications, the GGT1 gene sequence appears to have been 
517 modified to form various members of the GGT family at different chromosomal loci that

518 consist of duplications of the GGT-spacer sequence (Fig. 6b) [14].

\section{Discussion}

Data presented in this manuscript point to a process of initiation of de novo gene birth in primates that arises from an intergenic spacer sequence. This non-coding DNA sequence was evolutionarily situated between genes GGT1 and GGT5 in genomes of ancestral prosimian primitive primates but remained attached to the GGT1 gene after the large primate genomic expansions. Its sequence has been conserved during primate evolution. Examples are provided that show varied sequences and diverse genes stem from the GGT1-spacer 3' end, or from a duplicated spacer sequence. The data point to the spacer as a nucleation factor for initiation of new gene sequences, with the FAM247 sequence consistently serving as the starting sequence.

FAM247, whose 5' side makes up the entire human GGT5 exon 1 sequence, appears to also be present in the zebrafish GGT5 exon 1, based on conserved amino acid analyses. Other data show that the 3 ' end of the human FAM247, a sequence, which forms exon 11 and the 3' UTR of the ubiquitin specific peptidase 18 (USP18) gene transcript [15] is present in zebrafish [18]. Thus, sections of the FAM247 sequence have been present in an early ancestor approximately 300 million years ago. The primate GGT5 gene appears to be descendant from an early ancestor, such as zebrafish, and GGT5 may have initially been born from a spacer sequence starting with an FAM247 type sequence in zebrafish or another ancestor. However, in terms of how the GGT5 gene sequence was elongated and completed, this is difficult to determine with current

543 data.

As the FAM247 sequence formed parts of genes and functional elements during evolution, it would be unusual if this sequence was an isolated example. There should be other sequences that formed parts of multiple, diverse genes and/or functional elements in different life forms during evolution, as well as the presence of other spacertype sequences.

A model is presented to show how the long non-coding RNA gene, $B C R P 3$ is born in the Rhesus monkey. The process consists of the initiation of sequence growth by the spacer using the FAM247 sequence, the elongation of the FAM247 sequence, followed 
554 by addition of a complex of tandem transposable elements and ending with the transfer

555 of a copy of the $B C R$ gene segment to the newly formed sequence.

The baboon, which together with the Rhesus monkey is part of the Old World monkeys, and the orangutan that is a part of the hominoids (great apes) appear to have both come to a dead end in BCRP3 development and did not progress to the extent of the Rhesus in BCRP3 sequence maturation. Both primate species show a more limited BCRP3 sequence. In addition, the partly formed BCRP3 sequences in the baboon and orangutan genomes have no known or predict genes stemming from the partial sequences. The gibbon only formed a partial FAM247 sequence at its GGT1-spacer locus and with no annotated genes predicted to be encoded within the sequence. These examples suggest a trial and error process in BCRP3 and FAM247 sequence maturation for these species. The final formation of the $B C R P 3$ gene in humans suggests a long-term evolutionary process involving gene development. Guerzoni and McLysaght [11] previously described the de novo formation of primate protein genes over evolutionary time; thus, the process of long term gene development during evolution may have occured with both protein and non-coding RNA genes.

Interestingly, the chimpanzee developed both the complete BCRP3 and FAM247 sequences and with a high identity of both sequences with the human gene sequences, but these sequences were formed at different chromosomal loci from those found in humans or the sites of synteny. This leaves the unanswered question of how the FAM247 gene sequence was formed in humans, i.e., by inheritance of the FAM247 the sequence from the chimpanzee followed by translocation of the sequence, or by de novo formation of the FAM247 sequence at the human locus having a GGT-spacer sequence and the RNA transcriptional apparatus to form an FAM247 RNA transcript.

The TE ALU/LINE repeats of the BCRP3 sequences have similarities to chromosomal satellite sequences, e.g., HSAT1, an element that was originally found on the $Y$ chromosome but is also present but abundantly found on chr22 [25-27]. How the tandem TE repeats that are present in BCRP3 originated in each primate species is not known. However, McGurk and Barbash [28] have pointed out that formation of tandem arrays may begin as TE dimer insertions followed by expansion to a tandem array. Also of interest are models for the birth of genomic satellite DNA repeats [29], which may pertain to the Alu/LINE TE tandem array seen here.

We do not know the function of the Alu/LINE TE tandem arrays. With centromere and pericentromeric satellites, some play a role in heterochromatin formation in Drosophila 
chr 22, which may be relevant. Other and diverse roles of satellites have been outlined

594 [31].

595

The evolutionary formation of the $B C R P 3$ sequence and gene presents a sharp contrast to the creation of the gene linc-UR-UB, the regulatory long non-coding RNA gene found in the human genome and believed to be involved in immune system regulation and formed by a simple transcriptional read through process [15]. This reiterates the wealth of mechanisms that life forms have used to create new genes [1-17]. synthesis, or if there is a template involved is a "black box". However, Liang et al [32] studied DNA synthesis with a thermophilic restriction-endonuclease-DNA polymerase and described DNA synthesizes without a template or primer; a role in the development of genes during early evolution was hypothesized. In addition, it was shown that a hyperthermophilic archebacterial DNA polymerase can elongate palindromic and imperfect palindrome tandem repetitive DNA [33]. The FAM247 5' end sequence begins with a small imperfect palindrome; the sequence then continues to approximately 2000 bp with sections of repetitive base pairs, and then is followed by TEs (S12 Fig.). With the $B C R P 3$ gene, which has part of the FAM247 sequence, the imperfect palindrome lies within the spacer sequence as the FAM247 sequence within BCRP3 starts at bp position 33 bp of FAM247 and positions 1-32 bp are within the spacer. Can this suggest template free elongation of FAM247 synthesis? Experimental studies are needed, and the significance the of FAM247 5' end imperfect palindrome needs to be assessed.

\section{Methods}

\section{Primate species genomes:}

620 Genomic sequences of species listed were accessed using Home gene NCBI:

621 (https://www.ncbi.nlm.nih.gov/gene) and BLAST Local Alignment

622 (https://blast.ncbi.nlm.nih.gov/BlastAlign.cgi)

\section{Species}

625 Humans, Homo sapiens (NCBI:txid9606)

626 Chimpanzee, Pan troglodytes (NCBI:txid9596)

627 Orangutan, Pongo abelii (:Sumatran orangutan) (NCBI:txid9601)

628 Baboon, Papio anubis (olive baboon) (NCBl:txid9554)

629 Rhesus, Macaca mulatta (Rhesus monkey) (NCBI:txid9544)

630 Tarsier, Carlito syrichta (Philippine tarsier) (NCBI:txid1868482) 
631 Lemur, Microcebus murinus (gray mouse lemur) NCBI:txid30608)

632 Opossum, Monodelphis domestica (gray short-tailed opossum) (NCBI:txid13616)

633 Mouse, Mus musculus (house mouse) NCBI:txid10090)

634 Zebrafish, Danio rerio (zebrafish) (NCBI:txid7955)

Gene source

637 The NCBI/NLM data base was the source of the chromosomal locations of genes, gene

638 annotations and gene sequences of primate and other species, Website: home gene

639 NCBI, https://www.ncbi.nlm.nih.gov/gene

\section{Nucleotide and amino acid sequence alignment programs:} The EMBL-EBI sequence analysis program, Clustal Omega Multiple Sequence Alignment (https://www.ebi.ac.uk/Tools/msa/clustalo/) [34 ] was primarily used for alignments of nucleotide and amino acid sequences as well as determining identities between sequences. The identities represent only aligned sequences and do not including gaps sequences. It should be pointed out that the percent identities can vary in comparisons of homologs with lower similarities.

Pairwise Sequence Alignment, EMBOSS Stretcher (https://www.ebi.ac.uk/Tools/psa/emboss_stretcher/) and EMBOSS Needle[34] were employed for aligning two sequences.

\section{Transposable elements and simple repeat analyses} employed to determine the TE Alu/LINE repeat sequences. Both search engines rm BLAST and AB-BLAST were used. Minor differences between results from both search engines did not affect the results or conclusions. An additional related resource is the Dfam data base [35], the data base for repetitive DNA families. It should also be pointed out that there are can ambiguities in annotation of TE subfamilies [36], this was not a problem in comparing TE patterns from different species. Dr. Jessica Storer, Institute for Systems Biology, provided TE and repeat sequence data present in the BCRP3 sequence using an updated RepeatMasker program.

\section{RNA expression}

666 www.ncbi.nlm.nih.gov/gene/, human tissue-specific expression from the New Transcript 667 table subfamilies [19].

\section{Availability of additional data on websites}

673 www.ncbi.nlm.nih.gov/gene/ 
HUGO Gene Nomenclature Committee: Home:

https://www.genenames.org

Additional database for gene properties:

GeneCards-the human gene database: (www.genecards.org)

680 HGNC: (Genenames.org)

Genes and expression-site guide:

683 https://www.ncbi.nlm.nih.gov/guide/genes-expression/

\section{Supporting information}

S1 Fig. a. Sequence alignment of the (GGT1), RefSeqGene with the GGT1-spacer sequences from mouse and primate species. b. Alignment of sequence from the Microcebus murinus (gray mouse) lemur with part of the 3' end sequence the (GGT1), 690 RefSeqGene sequence.

691 S2 Fig. Alignment of complete sequences from spacers.

692 S3 Fig. Amino Acid sequence alignment of GGT5 from various species

693 S4 Fig. The alignment of the BCRP3 sequence present in the Rhesus locus that

694 contains the sequence between LOC106996293 and GGT1 and human BCRP3 gene.

695 S5 Fig. Alignment of the BCRP3 sequence from the baboon with the BCRP3 gene and 696 Rhesus BCRP3 sequences.

697 S6 Fig. Alignment of the gibbon sequence between GGT2 and GGT1 with the human 698 BCRP3 sequence.

699 S7 Fig. Alignment of the gibbon sequence between GGT2 and GGT1 with the human 700 BCRP3 sequence.

701 Nucleotide sequence alignment of the orangutan sequence that contains the BCRP3 702 sequence, with the human BCRP3 gene sequence.

703 S8 Fig. The orangutan tandem TE repeat array showing three SVA_A insertions. Data 704 kindly provided by Dr. Jessica Storer.

705 S9 Fig. Alignment of the chimpanzee sequence between genes LOC112206721-

706 LOC112206738 (containing the GGT1-spacer duplication locus) with the human

707 BCRP3.

708 S10 Fig. Alignment of sequence between genes LOC112206721-LOC112206738 in

709 chimpanzee with human BCRP3.

710 S11 Fig. Alignment of the chimpanzee sequence between GGT1 and LOC749026 with 711 the FAM247A sequence in humans. 
712 S12 Fig. FAM247 5' end imperfect palindrome and repeats,

714 Acknowledgements

I am grateful to Dr. Jessica Storer, Institute for Systems Biology, Seattle, Washington

References

2. Ohno S. Gene duplication and the uniqueness of vertebrate genomes circa 19701999. Semin Cell Dev Biol. 1999;10(5):517-522. doi:

10.1006scdb.1999.0332.PMID: 10597635

3. Korbel JO, Kim PM, Chen X, Urban AE, Weissman S, Snyder M, Gerstein MB. The current excitement about copy-number variation: how it relates to gene duplications and protein families. Curr Opin Struct Biol. 2008;18(3):366-374. doi: 10.1016/j.sbi.2008.02.005. PMID: 18511261 dynamics of adaptor protein complex subunits in embryophytes. Traffic. 2019; 20(12):961-973. doi: 10.1111/tra.12698.

6. Long M, Betrán E, Thornton K, Wang W. The origin of new genes: glimpses from the young and old. Nat Rev Genet. 2003;4(11):865-875. doi: 10.1038/nrg1204.

736 PMID: 14634634

7. Levine $M T$, Jones $C D$, Kern AD, Lindfors HA, Begun DJ. Novel genes derived from noncoding DNA in Drosophila melanogaster are frequently X-linked and exhibit testisbiased expression. Proc Natl Acad Sci U S A. 2006; 103(26):9935-9939. doi: Genome Res. 2009;19(10):1752-1759. doi: 10.1101/gr.095026.109. Epub 2009 Sep 2. 
9. Wu DD, Irwin DM, Zhang YP De novo origin of human protein-coding genes.

748 PLoS Genet. 2011;7(11):e1002379. doi: 10.1371/journal.pgen.1002379. Epub 2011

Nov 10. PMID: 22102831

10. Carvunis AR, Rolland T, Wapinski I, Calderwood MA, Yildirim MA, Simonis N, Proto-genes and de novo gene birth. Nature. 2012;487(7407):370-4. doi:

753

754 10.1038/nature11184. PMID: 22722833

11.Guerzoni D, McLysaght A. De Novo Genes Arise at a Slow but Steady Rate along the Primate Lineage and Have Been Subject to Incomplete Lineage Sorting.

12. Luis Villanueva-Cañas J, Ruiz-Orera J, Agea MI, Gallo M, Andreu D, Albà MM. New Genes and Functional Innovation in Mammals. Genome Biol Evol. 2017;9(7):18861900. doi: $10.1093 / \mathrm{gbe} / \mathrm{evx136.}$

762

763

764

13. Liu WH, Tsai ZT, Tsai HK. Comparative genomic analyses highlight the contribution of pseudogenized protein-coding genes to human lincRNAs. BMC Genomics. 2017;18(1):786. doi: 10.1186/s12864-017-4156-x.PMID: 29037146

14. Delihas N. Formation of human long intergenic non-coding RNA genes, pseudogenes, and protein genes: Ancestral sequences are key players. PLoS One. 2020 Mar; 15(3):e0230236. doi: 10.1371/journal.pone.0230236. eCollection 2020. PMID: 32214344

15. Rubino E, Cruciani M, Tchitchek N, Le Tortorec A, Rolland AD, Veli Ö et al. Human Ubiquitin-Specific Peptidase 18 Is Regulated by microRNAs via the 3'Untranslated Region, A Sequence Duplicated in Long Intergenic Non-coding RNA Genes Residing in chr22q11.21. Front Genet. 2021 Feb 3;11:627007. doi: 10.3389/fgene.2020.627007. eCollection 2020. PMID: 33633774

16. Shiao MS, Khil P, Camerini-Otero RD, Shiroishi T, Moriwaki K, Yu HT. Origins of new male germ-line functions from X-derived autosomal retrogenes in the mouse. Mol Biol Evol. 2007;24(10):2242-53. doi: 10.1093/molbev/msm153. PMID: 17646254

17. Stewart NB, Rogers $R L$ Chromosomal rearrangements as a source of new gene formation in Drosophila yakuba. PLoS Genet. 2019;15(9):e1008314. doi: 10.1371/journal.pgen.1008314. eCollection 2019 Sep. PMID: 31545792

18. Delihas N. Genesis of Non-Coding RNA Genes in Human Chromosome 22-A Sequence Connection with Protein Genes Separated by Evolutionary Time. 
19. Fagerberg L, Hallström BM, Oksvold P, Kampf C, Djureinovic D, Odeberg J. et al. Analysis of the human tissue-specific expression by genome-wide integration of transcriptomics and antibody-based proteomics. Mol Cell Proteomics. 2014. 13, 397406. 10.1074/mcp.M113.035600

20. Zinner, D. Keller, C. Nyahongo JW, Butynski TM, de Jong YA, Pozzi, L. Distribution of mitochondrial clades and morphotypes of baboons Papio spp. (Primates: Cercopithecidae) in eastern Africa. J. East African Nat. Hist. 2015. 104, 143-168. doi.org/10.2982/028.104.01.

21. Savage AL, Bubb VJ, Breen G, Quinn JP. Characterisation of the potential function of SVA retrotransposons to modulate gene expression patterns. BMC Evol Biol.

22 Vogt J, Bengesser K, Claes KB, Wimmer K, Mautner VF, van Minkelen R, et al. SVA retrotransposon insertion-associated deletion represents a novel mutational mechanism underlying large genomic copy number changes with non-recurrent breakpoints. Genome Biol. 2014;15(6):R80. doi: 10.1186/gb-2014-15-6-r80. PMID: 24958239

23. Babcock M, Pavlicek A, Spiteri E, Kashork CD, loshikhes I, Shaffer LG, et al. Shuffling of genes within low-copy repeats on 22q11 (LCR22) by Alu-mediated recombination events during evolution. Genome Res. 2003;13(12):2519-32. doi:

24. Wang Y, Liu F, Wang W. Dynamic mechanism for the transcription apparatus orchestrating reliable responses to activators. Sci Rep. 2012;2:422. doi: have we learned? Annu. Rev. Genomics Hum. Genet. 2009, 10, 355-386);

26. Kato T, Kurahashi H, Emanuel BS. Chromosomal translocations and palindromic

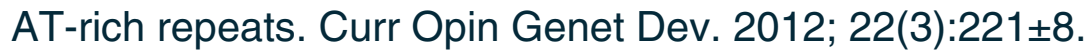
https://doi.org/10.1016/j.gde.2012.02.004 PMID: 22402448

27. Delihas N. A family of long intergenic non-coding RNA genes in human chromosomal region 22q11.2 carry a DNA translocation breakpoint/AT-rich sequence. PLoS One. 2018; 13(4):e0195702. doi: 10.1371/journal.pone.0195702.

PMID: 29668722

28. McGurk MP, Barbash DA. Double insertion of transposable elements provides a substrate for the evolution of satellite DNA. Genome Res. 2018; 28(5):714-725. doi: 10.1101/gr.231472.117. PMID: 29588362 
83629 Ahmad SF, Singchat W, Jehangir M, Suntronpong A, Panthum T, Malaivijitnond S, et 837 al. Dark Matter of Primate Genomes: Satellite DNA Repeats and Their Evolutionary

838 Dynamics. Cells. 2020; 9(12):2714. doi: 10.3390/cells9122714. PMID: 33352976. Significance of Satellite DNAs: Insights From Drosophila._Front Cell Dev Biol. 2020;8:312. doi: 10.3389/fcell.2020.00312. PMID: 32432114

31. Thakur J, Packiaraj J, Henikoff S. Sequence, Chromatin and Evolution of Satellite DNA. Int J Mol Sci. 2021; 22(9):4309. doi: 10.3390/ijms22094309. PMID: 33919233

32 Liang X, Jensen K, Frank-Kamenetskii MD. Very efficient template/primerindependent DNA synthesis by thermophilic DNA polymerase in the presence of a thermophilic restriction endonuclease. Biochemistry. 2004;43(42):13459-66. doi: 10.1021/bi0489614. PMID: 15491153]

33. Ogata N. Elongation of palindromic repetitive DNA by DNA polymerase from hyperthermophilic archaea: a mechanism of DNA elongation and diversification. Biochimie. 2007;89(5):702-12. doi: 10.1016/j.biochi.2006.12.011. PMID: 1732166

34. Madeira F, Park YM, Lee J, Buso N, Gur T, Madhusoodanan N, et al. The EMBLEBI search and sequence analysis tools APIs in 2019. Nucleic Acids Res. 2019;47(W1):W636-W641. doi: 10.1093/nar/gkz268. PMID: 3097679]

35. Storer J, Hubley R, Rosen J, Wheeler TJ, Smit AF. The Dfam community resource of transposable element families, sequence models, and genome annotations. has a reproducibility problem. Mob DNA. 2021;12(1):4. doi: 10.1186/s13100-021-00232- 


\section{Primate genomic expansion between GGT1 and GGT5}

Mus musculus (house mouse)

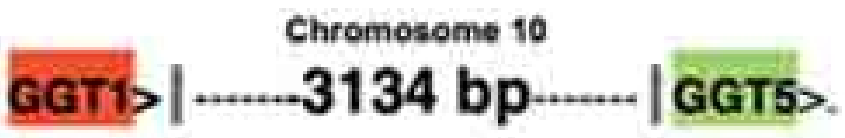

Carlito syrichta (Philippine tarsier)

$$
\text { GGTD } \mid \begin{gathered}
\text { Unplaced scattold } \\
-2872 \text { bp --.-| }
\end{gathered} \text { GGT5> }
$$

Macaca mulatta (Rhesus monkey)

Chromosome 10

\section{Pan troglodytes (chimpanzee)}

Chromosome 22 343,330 bp

Homo sapiens (human) 
a.

Mouse GGT1|—spacer-—। GGT5 gene

Tarsier GGT1|—spacer-_-

\section{GGT5 gene}

Rhesus

GGT1|---_spacer-1 - - TE repeatLOC107000612

(breakpoint cluster region protein-like) gene

Chimpanzee GGT1|—spacer-_genes LOC107973089-LOC112206779|

Human GGT1|—-spacer-— --TE repeat$B C R P 3$ gene

b. Segmental duplications in human chr22

GGT2|—spacer-_ FAM247A gene

GGTLC3|—spacer_—-|No gene expression but FAM247 sequence GGT3P|---spacer- $\mid$ FAM247C gene

GGTLC5P|-spacer_- FAM247D gene

\section{Figure 2}



GGT1.END-GGT5.end.75422027-75453034.mouse
GGT1.END-GGT5.START. $75422027-75425161$.mouse GGT1 end-GGT5 .beginining.Philippine.tarsier.ref FAM247.LOC105372935.ref.human GGT1 .end-FAM247.start. Rhesus.ref. GGT1 .end-start.BCRP 3. .human. ref GGT1end-LOC749026.end. 7456450-7520130.chimp GGT1.end-FAM247.start.chimp.ref

GGT1 .END-GGT5 .end. 75422027-75453034.mouse GGT1 .END-GGT5.START . 75422027-75425161.mouse GGT1 .end-GGT5.beginining. Philippine.tarsier.ref FAM247.LOC105372935.ref.human GGT1 .end-FAM247. start. Rhesus .ref. GGT1 .end-start. BCRP 3. .human. ref GGT1end-LOC749026.end.7456450-7520130.chimp GGT1 .end-FAM247. start.chimp.ref

GGT1 .END-GGT5 .end. 75422027-75453034.mouse GGT1.END-GGT5.START . 75422027-75425161.mouse GGT1 end-GGT5 beginining. Philippine.tarsier .ref FAM247.LOC105372935.ref.human GGT1 .end-FAM247.start. Rhesus .ref. GGT1 .end-start. BCRP 3. . human. ref GGT1end-LOC749026.end. 7456450-7520130.chimp GGT1 .end-FAM247. start.chimp.ref

GGT1 .END-GGT5.end. 75422027-75453034.mouse GGT1 .END-GGT5.START . 75422027-75425161.mouse GGT1 end-GGT5 .beginining. Philippine.tarsier.ref FAM247.LOC105372935.ref.human GGT1 .end-FAM247. start. Rhesus .ref. GGT1 .end-start. BCRP 3 . . human. ref GGT1end-LOC749026.end. 7456450-7520130.chimp GGT1.end-FAM247.start.chimp.ref catcacatttccaatggcactgggactgaggagtctttgggtggtgttggggcagcaggg catcacatttccaatggcactgggactgaggagtctttgggtggtgttggggcagcaggg cgccaaggcctcaagcatattcagcggggatgggac-------------------

caccaagttctcctgcacattgggacagtgtgacctgggctctggttagtg--gcaggt caccaagttctcctgcacattgcgacagtgtgaccetgggctctggcgggca--gtaggt caccaagttctcctgcacattgcgacagtgtgaccetgggctctggcgggcg--gtaggt caccaagttctcctgcacattgcgacagtgtgaccetgggctctggcgggcg--gtaggt

caggccatgggatcaactggcgatggaagagttaacagcggcagctggctcttctcaaga caggccatgggatcaactggegatggaagagttaacagcggcagctggctcttctcaaga -------------cacggcagcaagggagttaaccgcagcagctggctc--ctgta-g

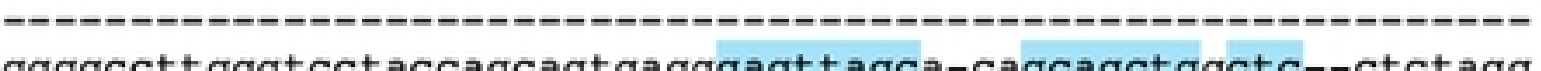
ggggcetttggacctaccagcagtgagggagttaaca-cagcagctgactc--ctctagg ggggcetttggacctaccagcagtgagggagttaaca-cagcagctgactc--ctctagg ggggcctttggacctaccagcagtgagggagttaaca-cagcagctgactc--ctctagg

\section{Start of mouse GGT5 gene sequence, highlighted in red}

aaaaaaaactcctgtaga--------tgcctggcttgcctccagggttgagcctcggg aaaaaaaactccetgtaga---------tgcctggett---------------------caaagaaaactcccc-cagacgetttgctgectggcettccgccagggetgagaa--cag

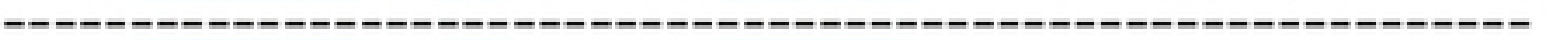
gaaggaaaactccettcagacactttggtgcctggcctcctgccaggaacaagca---gg caaggaaaactccctcagacgctttgctgcctggcetcctgccagcaacaagca---gg caaggaaaactccctcagatgctttgctgcctggcetcctgccagcaacaagca---gg caaggaaaactccctcagatgctttgctgcctggectcctgccagcaacaagca---gg

\section{Start of FAM247 sequence, highlighted in green}

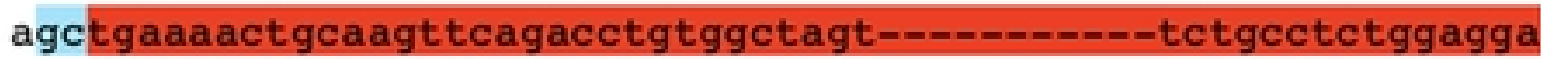
-----------------------------------------------------ggctgaaaactggaagttgaggegtgagcatagcacactctccetccgaagtgagcgett ---tgaaaactagaagttgaggcatgagtttggc-------cactccgtagtgtgcactt agctgaaaactagaagttgaggcataagtttggc-------c-----------------agctgaaaaccagaagttgaggegtgagtttggt-------ca--------------agctgaaaactagaagttgaggegtgagtttggc-------cactccgtagtgtgcactt agc- 
Percent Identity Matrix - created by clustal2.1

1: 1.exon1.GGT5.zebrafish 39.22

2: 5.exon1.GGT5.opossum

46.43

3: 4.exon1.GGT5.mouse 70.91

4: 1.exon1.GGT5.human 100.00

5: 2.exon1.GGT5.Rhesus.

96.49

6: 3.exon1.Philippine.tarsier

84.21

CLUSTAL O(1.2.4) multiple sequence alignment

exon1.GGT5b.zebrafish 5. exon1.GGT5. opossum 4. exon1.GGT5.mouse 1. exon1.GGT5.human 2. exon1.GGT5.rhesus. 3. exon1.Philippine.tarsier
MAKSQSRRCCFCLLALVC--TAAIICICILFSK-----QKCDFTRAAVSADSLMCSDIGR 53 MARPGGRAVCLILLAAGL--LAAIIAAACTLGRAAATCPAASYRTAAVAADTPRCSAIG- 57 MAWGHRATVCLVLLGVGLGL--VIVVLAAVLSPRQASCGPGAFTRAAVAADSKICSDIG- 57 MARGYGATVSLVLLGLG--LALAVIVLAVVLSRHOAPCGPQAFAHAAVAADSKVCSDIG- 57 MARGCGATVGLVLLGLG--LALAVIVLAVVLSRHQAPCGPQAFAHAAVAADSKVCSDIG- 57 MAWGCRAI ISLVLLGLGLGLALVI IVLAVVLPRHQAPCGPQAFAHAAIAADSKVCSDIGR 60 ** $\quad$ : * : * . : : $\quad$ :

Figure 4 


\section{a. Components of the partial BCRP3 sequence in the Rhesus monkey compared to human $B C R P 3$ gene}

\section{Human}

BCRP3 gene chr22

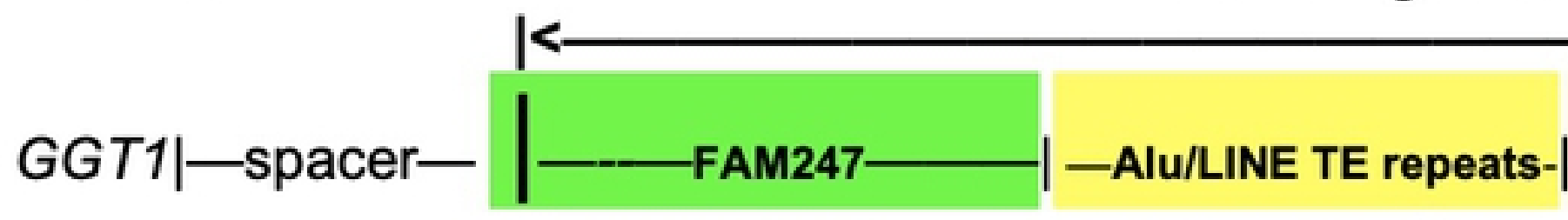

-BCR 3'end

-9959 bp of human BCR

bioRxiv preprint doi: https://doi.org/10.1101/2022.01.12.475983; this version posted January 12, 2022. The copyright holder for this preprint (which was not certified by peer review) is the author/funder, who has granted bioRxiv a license to display the preprint in perpetuity. It is made

Rhesus

BCRP3 sequence chr10

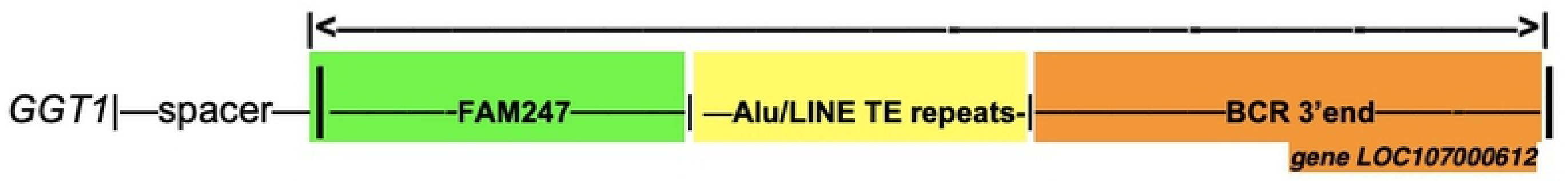

\section{b. Proposed formation of BCRP3 sequence in Rhesus}

1. Initiation and growth of FAM247 sequence

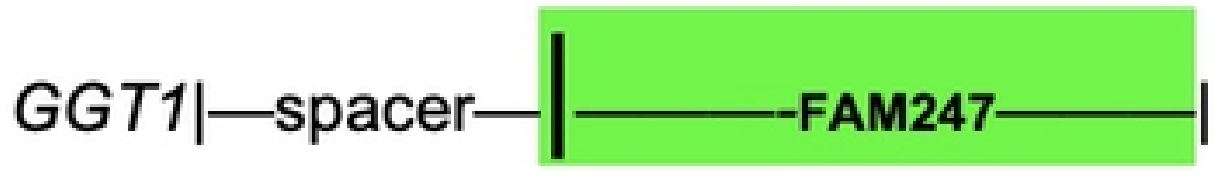

2. Addition of Alu/LINE TE repeats

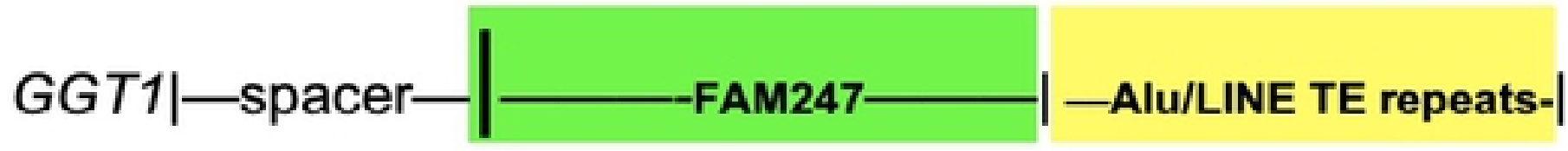

3. Addition of BCR 3' segment

GGT1|-spacer-| FAM247 - -Alu/LINE TE repeats-| BCR 3'end

Figure 5 


\section{a.}

Gibbon

BCRP3 sequence (complete sequence)

GGT2. |spacer| 5960 bp FAM247-Alu/LINE TEs

LOC115835989 breakpoint cluster region protein-like LOC115835847 the, putative POM121-like protein 1

\section{Orangutan}

BCRP3 sequence (incomplete sequence)

GGT1 |spacer| | 5960 bp FAM247-Alu/LINE TEs| no BCR sequence

$$
\begin{aligned}
& \text { (3 SVA_A } \\
& \text { insertions } \\
& 2 \text { LiMEg } \\
& \text { deletions }
\end{aligned}
$$

\section{Chimpanzee}

\section{Sequence}

|-.-partial GGT___ spacer| BCRP3 complete sequence

\section{Annotation}

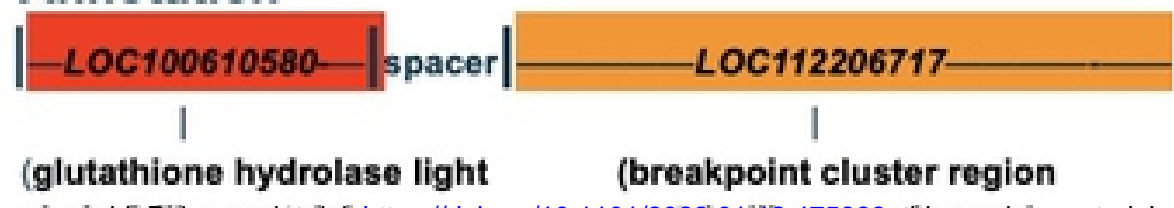

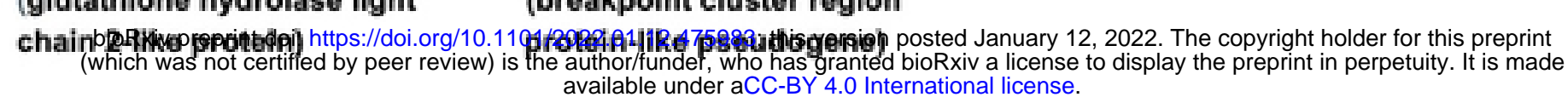

Human (BCRP3 gene at chr22 LCR22H)

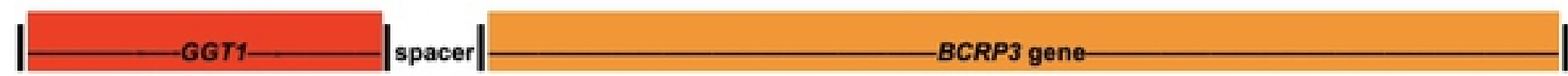

b.

Gibbon

incomplete FAM247 sequence

\section{Chimpanzee}

\section{Sequence}

|_GGT1_____ spacer|___ FAM247 complete sequence__

\section{Gene annotations}

$\mid$ |

(protein genes)

\section{Human chr22 LCR22D}

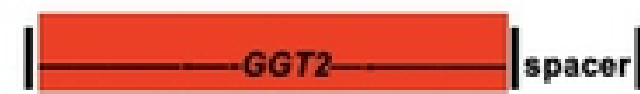

FAM247A gene

\section{Human Unlocalized Scaffold region of human chr22 ( NT_187386.1)}

inactive glutathione hydrolase 2 protein

\section{FAM247 complete sequence}

(no gene annotations)

\section{Figure 6}

